means full rest is another question. Dues it mean rest for the ligaments and capsules which are stretched? Does it favour the absorption of fluid or the various processes by which nature effects a cure? No. Take, for instance, adhesions. Does it favour them? There is no one who will not acknowledge that adhesions are often salutary. They limit inflammatory processes and they encapsule irritants, they give fixation to parts to which movement would be harmfal. No ; turn it how one will extension by weight and pulley leaves much to be desired.

My next point is that osteotomy brings on the scene a very beneficent factor. This is a question on which I particularly ask your opinion, for while this factor is for me a threadbare clinical experience its very existence is, I know, doubted by one eminent authority. Is it or is it not a fact that consequent on the section of bone there occurs in the neighbourhood of the point of section an alteration of the bone's economy which has, as a rale, a salutary effect on any taberculous inflammation there existing? I say it is a fact. An occasion on which I saw this strikingly manifested was one where I proceeded to excise a knee in a case of long standing tuberculous disease without having got leave from the patient to perform an amputation should occasion require it. As soon as $I$ had removed the articular surfaces I found that the disease extended so far in both bones that its removal would have made union impossible. I therefore contented myself with approximating and fixing the parts. Without further surgical interferences these bones in which for years a rarefying osteitis had been advancing and in which prior to operation there had been apparently no $\in$ fforts at repair healed thoroughly and made for the patient a useful limb. But why weary the reader with every-day incidents?

On the fact which they teach-viz, that tuberculous bone tends to mend after being incised-Kirkpatrick, Stoker, Stokes, and Macnamara have founded methods of treatment which have yielded good results. Almost my first experience of the Royal Academy of Medicine in Ireland was a discussion in the Section of Surgery on this subject raised by interesting papers from Sir Thornley Stoker and Sir William Stokes. On that occasion the Section was almost unanimous in agreeing with their views. For myself $I$ am in entire agreement with them and $I$ have followed their advice in more than one case with good results. It is, however, a question how incision or resection of bone does its beneficent work. Some say by the drainage it affords. The explanation which occurs to me is rather something of this sort. Under ordinary circumstances there is in tuberculous bone little or no tendency to bony reparation, one sees no osteophytes thrown out or other indications of reactive vitality. Resection of the bone excites this vitality. It calls upon it to put forth its whole strength for the repair of the injury. There is a new régime before which the tuberculous process has to yield-a régime which in the case of a fracture passing from the great to the lesser trochanter must extend somewhat to the neck and head of the femur. Is it not the case that in examining a specimen of recently united fracture in any bone one sees a more or less sclerosed area extending well beyond the scene of injury and that this condition is the very reverse of that which one meets with in spreading tuberculous disease? Anyhow, whatever the explanation, the clinical fact remains and the osteotomy I suggest gives a large number of patients with tuberculous hips the benefit of it, since most observers are in agreement that in the hip the usual situation of primary trouble is at the lower part of the neck of the femur jast outside the epiphyseal cartilage.

The most serious objection which I have seen urged against this plan of treatment is by Mr. Owen in the "Yearbook of Treatment," to the effect that either non-union will follow the osteotomy or that union will take place at such an angle that the inevitable shortening becomes greatly intensified. With these objections experience must deal. As yet it is altogether favourable to the operation, but of course siz cases are too few to prove anything. It should, in considering the danger of non-union, be remembered that after straightening the limb the fragments still in apposition are not further disturbed, the soft parts are not torn and the limb is at once placed in a splint. The same consideration-i.e., the fact that the force which breaks the bone does not displace the fragmentstaken in conjunction with the shortness of the upper fragment, will explain the very slight degree of shortening which has as yet occurred in these cases. As regards treatment, when the patient is up and about I hold by Thomas's splint. I use it from the outset with patients who present themselves early, for it is easily fitted to a fairly straight limb, but for straightening a flexed and adducted limb I prefer an osteotomy. The splint should be worn for a considerable time. No treatment can give quickly to a diseased hip the strength necessary to bear the weight of the body. One way may be less slow than another, but all must be slow. A want of consideration of this point may cause new mothods to be unjustly tried. It should not, however, make the surgeon rest content with things as they are. No one will convince me that the treatment of hip-joint disease has reached its highest development. Its present position is one calling loudly for the attention of the general surgeon, for since the surgeon can not in general hos pitals detain hip' cases as long as is desirable it is specially incumbent on him to do as much as possible for them during the period of their stay and to discharge them in as forward a state as possible. Dublin.

\section{THE ADMINISTRATION OF LARGE DOSES OF GUAIACOL IN PHTHISIS.}

BY J. RDWARD SQUIRE, M.D., M.R.C.P. LoND., D.P.H. CAMB.,

PHYSICIAN TO THE NORTH LONDON HOSPITAL FOR CONSUMPTION.

THE accompanying report, which has been drawn up for me by Mr. C. Stanford Read, the resident medical officer at the North London Hospital for Consumption, is, I think, sufficiently interesting to publish. It is, of course, incomplete, as the observations are still in progress ; but it establishes the fact that patients can take pure liquid guaiacol in doses of one drachm three times daily (180 minims in the day), not only without toxic effects, but apparently with decided benefit. Creosote has been given in doses of from 160 to 180 minims a day and carbonate of guaiacol in doses of from 45 to 60 grains three times a day, but I know of no record of guaiacol itself approaching these quantities. The amount of guaiacol in creasote is somewhat indefinite; the carbonate is said to contain about 91.5 per cent. of pure guaiacol. A drachm of carbonate would thus be equal to about 55 grains of pure guaiacol. The liquid guaiacol is not quite pure, so that the amount of guaiacol taken will be much the same with a similar dosage of the carbonate or of the liquid guaiacol. The latter has the advantage of being less expensive. The caustic action of uncombined guaiacol on the mucous membrane and some fear of toxic effects have prevented the prescribing of full doses of this drag, but it will be seen from the following report that with due care in administration most patients can safely take half a drachm, or even one drachm, three times a day. The drug was given in capsules containing 5 minims each, or in emulsion with glycerine and tincture of orange peel, and was always followed by a drink of milk. The doses were taken after meals. I have not attempted to exceed the dose of 60 minims, or to give more than 180 minims in the twenty-four hours, but some of the patient:s have continued taking this amount for several weeks with apparent benefit. One patient who has recently been discharged after taking this quantity for three weeks gained about $1 \frac{1}{2} \mathrm{st}$. during the three and a half months that he was in hospital and left in a condition which might well be described as "cured." Short notes of this case will be found at the end of this report. It has been stated ${ }^{1}$ that after large doses of gaaiacol the urine gives a precipitate with hydrochloric acid. I am unable to endorse this statement, for the urine of patients taking large doses of the drug has not given any precipitate with hydrochloric acid in those cases which I have tested.

Report on the treatment of phthisis by guaiacol, by $\mathrm{Mr}$. C. STANFORD READ. - Guaiacol bas often been administered in the North London Hospital for Consumption in certain cases in the past, usually in capsules of 5 minims each, after the three principal meals of the day. This has bitherto been done in cavitation cases accompanied by profuse or fotid expectoration with very good results as far as this one particular symptom went. Of late, at the suggestion of Dr. Squire, we have pushed the

1 Eschle. Quoted by Dr. R. Seifert, Thr Lancer, Nov. 14th, 1896. 
drag in all stages of the disease to see how much patients could take without ill effect and what good results, if any, would follow. In many cases after starting the treatment one has been obliged to cease the administration of the drug as other symptoms or complications (not connected with the guaiacol) have arisen necessitating its withdrawal. We have nevertheless 40 patients who have taken the drug: 6 cases took 60 minims, 2 cases took 50 minims, 4 cases took 40 minims, 6 cases took 30 minim $\%, 10$ cases took 20 minims, 6 cases took 15 minims, and 6 cases took 10 minims three times a day after food. Some of these are still in hospital and are gradually increasing the dose. The drug was first administered in capsules, each containing 0 minims, but later was given in an emulsion with glycerine and tincture of orange peel, many patients taking it partly in one form and partly the other. A good few found difficulty in swallowing many capsules; others, again, preferred them much to the acrid liquid; in either case they would drink about half a cupful of milk with the guaiacol, which seemed to greatly add to its being borne well. The dose of 5 minims was at first usually increased by 5 minims every third day, and later more rapidly, till 60 minims were reached. Only one patient felt any ill effects; he, when taking 20 minims three times a day, complained of much stomachic and abdominal pain with a sinking sensation in the epigastrium, passing a small quantity of blood by the bowel. The guaiacol was stopped, but resumed later with 10-minim doses withont ill effect. 26 were cavitation cases and in these the diminution in the amount of expectoration was very marked, beginning to diminish early in the administration of the drug. Some patients whose expestoration was very profuse on admission would later only cough up little "pellets" of sputum first thing in the morning and none after. There bas been no marked effect on the tempsrature observed when guaiacol has superseded other drugs, but in those taking it continually a steady lowering of the evening temperature bas been commonly noticed. 35 of the cases put on weight, most of them well, one putting on $19 \mathrm{lb}$, in twelve weeks. There was not, as far as I could make out, any definite relation between the increase in weight and the proportional increase of the doses. The cough was in no special way affected that I could see, but night sweats in nearly all cases diminished and then disappeared in a very short space of time. 12 cases had laryngeal phthisis as well, but as these cases all had local treatment also one cannot say whether the guaiacol had any special effect or no. The patients complained a good deal either of the emulsion burning their throat or of their difficulty in swallowing the capsules, but by dint of perseverance combined with faith in the drug they managed very well after a time. The effect of the drug, if any, on the number, \&c , of the bacilli in the sputum is now being observed.

The following is an abstract of the case referred to in the former part of this paper. The patient was a man aged twenty - four years. He was admitted to hospital on Nov. 18th, 1897, complaining of much weakness, sickness, and cough. The patient had been working harder than usual of late and had got run down. The first symptom was hæmoptysis (one pint) six weeks before admission. Congh with weakness and wasting gradually supervened. On admission he was found to be a cachectic pale man. He was $5 \mathrm{ft} .4 \frac{1}{4}$ in. in height and weighed $8 \mathrm{st} .13 \mathrm{lb} .6 \mathrm{oz}$. He had very profuse night sweats and much sickness; the congh was not very troublesome; he had muco-purulent expectoration. With regard to physical signs, there was very slight impairment of the percussion note at the left apex, with a few sharp "clicks" at the end of inspiration. He had continuous temperature of about $102.4^{\circ} \mathrm{F}$. He was treated with an acid tonic and complete rest in bed. On Nov. 22nd the acid was omitted; quinine and digitalis were given. On the 25 th the temperature was gradually falling. He was still sweating much at night. On Dec. 5th the temperature had been remittent, but was continuously high again and was going up. The patient was sick a good deal. Large râles were now heard in the second, third, and fourth spaces in the left front and down the left back commencing from the apex of the lower lobe. On the 10th 2 minims of guaiacol three times a day after food were ordered. He had gradually lost weight up to now. He was ont of bed for an hour and could hardly stand. On Jan. 3rd, 1898, the patient was taking 15 minims of guaiacol three times a day. Fine râles were heard in the left front with some crepitations below. There were a few crepitations at the base in front. Râles heard at the back were much smaller. The temperature kept about $99^{\circ}$. He had put on $8 \mathrm{lb}$. in weight since getting up (Dec. 10 hh). On the 31 st the râles were gradually drying up. He was taking 35 minims of guaiacol three times a day. He had pat on 1 st. in weight. His temperature was normal. He complained of nothing except cough and expectoration the first thing in the morning. From Feb. 15th to 17th there was pain in the left base, friction was heard, and the chest was strapped. 1 drachm of guaiacol three times a day, all in capsules, was given with no ill-effects. His temperature was normal. He was 1 st. $5 \mathrm{lb}$. heavier than on Dec. 10th. On March 7th the patient went out. He expressed himself as perfectly well and strong. He had taken 1 drachm of guaiacol thrice daily up to now. He had gained $22 \mathrm{lb}$. in weight since Dec. 10th, when he commenced guaiacol. There were no tubercle bacilli in the sputa when he left the hospital.

Harley-street, W.

\section{A THEORY OF NERVOUS CONDUCTION.}

By W. S. HEDLEY, M.D. EdIN., M.R.C.S. ENG.

IN 1889 it was observed by Professor Oliver Lodge that two knobs sufficiently close together could, when a spar passed between them, actually cohere and, with a single voltaic cell in circuit, conduct "an ordinary bell-ringing current." Shortly afterwards M. Edouard Branly ${ }^{1}$ found that a tube of metallic filings enormously diminished its resistance when exposed to the neighbourhood of a Leyden jar or coil sparks. An arrangement of this kind proved to be a sensitive detector of radiation and such discontinuous conductors came to be called "coherers." The cohesion brought about by electrical means could be broken down by mechanical means. Thus sound-vibration or slight taps would restore the contact to its original condition of high resistance.

In a paper presented to the Académie des Sclences on Dec. 27th last M. Branly suggests certain points of possible resemblance between coherer action and the conductivity of the nerves for nervous impulses. He points out that in reality there is no very clear line of demarcation between continuous and discontinuous conductors; it is rather a question of degree. Passing from artificial to "natural" conductors he argues that the use of the term "nervous current" since the earliest days of physiological research seems to point to some recognised resemblance between nervous and electrical conduction. Until recently it was thought that the various elements of the nervous system were continuous. Now, since the advent of that trinity in unity known as the neurone the nerrous system may be regarded as composed of discontinuous elementsi.e., of elements contiguous but not continuous. It thus becomes possible to regard the neurone as the counterpart of the metallic granule of certain discontinuous conductors. As a blow rill weaken and eren abolish the conducting power in the latter, so traumatism may produce anæsthesia and hysterical paralysis-the latter due to a suppression of transmission, sensory or motor, of the nervous influence consequent on a defective contiguity of nerve elements. Again, as the oscillation of electrical discharges establishes the conductivity of discontinous conductors, so it is known that such discharges act efficiently in the cure of paralysis and hysterical anæsthesia. The possibility, therefore, sug gests itself that in both cases the effect is determined by bringing about the contignity of the elements of the conductor or some modification equivalent to contiguity. The parallelism between the action of a blow and of sparks upon discontinuous conductors and upon the hysterical nervous system may be carried further in the susceptibility common to both of reacting under a feeble stimulus when once a powerfal action is produced as a first effect-a condition which M. Branly has referred to in a former note to the Academy as " sensibilisation par un premier effet" (Dec. 6th, 1897). The high frequency discharges and the electric oscillations which accompany them are especially apt to make discontinuous conductors conduct, and it is such discharges that have been shown by d'Arsonval to have 